\title{
O crescimento de Parastacus defossus (Crustacea: Decapoda: Parastacidae)
}

\author{
Clarissa K. Noro \& Ludwig Buckup
}

\begin{abstract}
Programa de Pós-Graduação em Biologia Animal, Departamento de Zoologia, Universidade Federal do Rio Grande do Sul. Avenida Bento Gonçalves 9500, prédio 43435, sala 214, 91501-970 Porto Alegre, Rio Grande do Sul, Brasil.

E-mail: clarissanoro@hotmail.com; lbuckup@yahoo.com.br
\end{abstract}

\begin{abstract}
The growth of Parastacus defossus (Crustacea: Decapoda: Parastacidae). The present study investigates the growth of Parastacus defossus Faxon, 1898, a fossorial species known only from Brazil (state of Rio Grande do Sul) and Uruguay. The estimated growth curve was calculated for animals from the wild and for juveniles cultivated in the laboratory. The specimens were collected monthly between January, 2003 to August, 2005, in Lami region, municipality of Porto Alegre, state of Rio Grande do Sul, Brazil. The von BerTalAnfFy (1938) growth model was used to estimate the growth curve. To calculate the modal values from the frequency distributions the software PeakFit $4.12^{\circ}$ SeaSolve Inc., was employed. The growth curve for field animals is described by the equation: $L t=30,98\left[1-\mathrm{e}^{-0,0026(t+44,86)}\right]$ and for the juveniles cultivated in laboratory: $L t=19,29\left[1-\mathrm{e}^{-0,0048(t+37,14)}\right]$. It was shown that, under laboratory conditions, the growth was slower than in the field. By comparing the data of $P$. defossus with those of $P$. brasiliensis (von Martens, 1869), it was possible to verify a slower growth, both in laboratory as under field conditions. The longevity of the studied species (1198.7 days or 40 months) also showed to be lower when compared to P. brasiliensis.
\end{abstract}

KEY WORDS. Crayfish; growth curve; von Bertalanffy.

Parastacidae reúne os lagostins de água doce do hemisfério Sul, com representantes na Austrália, Tasmânia, Nova Zelândia, Madagascar e América do Sul, sendo que a maior parte das espécies ocorre na região australiana Na América do Sul, os Parastacidae estão representados por dez (10) espécies e três gêneros: Parastacus Huxley, 1879, Samastacus Riek, 1971 e Virilastacus Hobbs, 1991. Apenas Parastacus está representado no Brasil, por Parastacus pilimanus (von Martens, 1869), P. brasiliensis_(von Martens, 1869), P. varicosus Faxon, 1898, $P$. defossus Faxon, 1898, P. saffordi Faxon, 1898 e P. laevigatus Buckup \& Rossi, 1980 (vide Buckup 1999).

Aspectos da biologia das espécies brasileiras de parastacídeos foram estudados por Buckup \& Rossi (1980), Fries (1984), Fontoura \& Buckup (1989) e Buckup (1999). A primeira investigação sobre o crescimento de uma espécie de Parastacus no Brasil foi realizada por Fries (1984) que acompanhou o crescimento de uma coorte de $P$. brasiliensis nascida em laboratório. FonToura \& BUCKUP (1989) voltaram a estudar o crescimento de $P$. brasiliensis, aplicando o modelo de von BERTALANFFy (1938), acompanhando o crescimento de animais em sucessivas coletas em um arroio no município de Mariana Pimentel, Rio Grande do Sul.

As curvas de crescimento podem contribuir na compreensão dos diferentes padrões no ciclo de vida de uma espécie, que associados a outros processos como muda e reprodução, auxiliam no entendimento dos aspectos biológicos de uma população (SANTOS 1978). A determinação da curva de cresci- mento é de fundamental importância para a análise da estrutura populacional de uma determinada espécie, pois seus parâmetros podem fornecer informações referentes ao tamanho máximo atingido pelos animais, a idade em que este tamanho é alcançado e sobre a taxa de crescimento (VALENTI et al. 1987). Para os crustáceos, o estudo da curva de crescimento pode ser feito por meio de cultivo da espécie ou pelo acompanhamento das coortes etárias resultantes da distribuição de freqüências dos organismos em função do tempo (FonTeles-FilHo 1987). O modelo matemático proposto por vON BERTALANFFY (1938) tem se mostrado eficaz no ajuste dos pontos empíricos e tem sido usado com sucesso no cálculo do crescimento de diversos crustáceos (D’INCAO et al. 1993, FonTOuRA \& BUCKUp 1989, Sampaio \& Valenti 1996, Bueno et al. 2000, Noro \& Buckup 2003, Castiglioni et al. 2004, Dumont \& D’Incao 2004, Pinheiro \& Taddei 2005, Silva-Castiglioni et al. 2006, Boos Jr et al. 2006, Ferreira \& D'INCAO 2008).

O presente trabalho teve como objetivo o estudo do crescimento de $P$. defossus, uma espécie fossorial que constrói suas habitações subterrâneas em forma de galerias ramificadas e interligadas em terrenos baixos e alagadiços, de ocorrência registrada apenas no Brasil (Rio Grande do Sul) (Buckup \& Rossi 1980) e no Uruguai (Zolessi \& PHilippi 1995). A curva de crescimento foi calculada para populações amostradas em seu habitat natural e comparada com o crescimento de juvenis cultivados em laboratório. 


\section{MATERIAL E MÉTODOS}

Espécimes de $P$. defossus foram coletados uma vez por mês, de janeiro de 2003 a agosto de 2005, em uma propriedade rural particular na região do Lami, município de Porto Alegre, Rio Grande do Sul. Cerca de trinta (30) exemplares foram extraídos em cada operação mensal de coleta do interior das galerias com o auxílio de uma bomba de sucção em PVC. Em laboratório os animais foram medidos quanto ao comprimento da carapaça (CC) com paquímetro digital com precisão de $0,01 \mathrm{~mm}$.

Os dados de crescimento dos juvenis em laboratório foram obtidos pelo acompanhamento de uma coorte eclodida em dezembro de 2003, de uma fêmea ovígera coletada em outubro de 2003. A fêmea, com 30,32 mm de comprimento, abrigava 35 ovos na região abdominal. Após a eclosão dos ovos, os juvenis foram cultivados e mensurados mensalmente durante um período de 26 meses (até fevereiro de 2006), quando restou apenas um indivíduo vivo desta coorte. Os juvenis foram cultivados em temperatura constante de $20^{\circ} \mathrm{C}$, fotoperíodo de 12 horas luz/12 horas escuro e alimentados com ração para peixes de fundo da marca Alcon três vezes por semana.

O crescimento em comprimento de $P$. defossus foi estimado pela análise da distribuição de freqüência de comprimento da carapaça em intervalos de classe de $2 \mathrm{~mm}$ ao longo do ano e conseqüente reconhecimento do deslocamento modal em função do tempo. Visando aumentar o valor representativo das unidades amostrais, optou-se pelo agrupamento trimestral correspondente às estações do ano.

Para a identificação dos valores das modas foi utilizado o software estatístico PeakFit 4.12@ SeaSolve Inc., que contém um algoritmo capaz de estimar as distribuições Gaussianas ou normais contidas nas freqüências dos grupos de comprimento, identificando um parâmetro central ("Peak", a1 da amplitude Gaussiana) que pode ser entendido como correspondente a moda. Para a escolha dos parâmetros centrais e traçado gráfico inicial da curva de crescimento, priorizou-se os pontos (picos, "peaks") com menor erro padrão e maior valor do coeficiente de determinação.

Para a análise do crescimento em comprimento foi utilizado o modelo de von BertalanfFy (1938): $C_{t}=C_{\infty}\left[1-e^{-k(t-t o)}\right]$, onde $\mathrm{C}_{\mathrm{t}}=$ comprimento da carapaça na idade $\mathrm{t}$; $\mathrm{C}_{\infty}=$ comprimento médio máximo da carapaça; $\mathrm{e}=$ base dos logaritmos naturais; $\mathrm{k}=$ constante relacionada à taxa de crescimento; $\mathrm{t}_{0}=$ parâmetro relacionado com o tamanho do animal ao nascer; $\mathrm{t}=\mathrm{a}$ idade considerada.

A longevidade ou idade máxima (tmax) foi calculada segundo dois métodos: utilizando a fórmula inversa de Bertalanffy (segundo Pinheiro \& Taddei 2005): tmax = t0 - (1/k) ln [1 $\left(\mathrm{Cmax} / \mathrm{C}_{\infty}\right)$ ] e pelo método de Pauly (1980): $\operatorname{tmax}=3 / \mathrm{k}+\mathrm{t} 0$, onde, k é a constante de crescimento; t0, idade em anos estimada pela equação de Bertalanffy; Cmax, tamanho do maior animal registrado durante o período estudado, $\mathrm{C}_{\infty}$ o tamanho assintótico.

\section{RESULTADOS}

\section{Lagostins coletados em campo}

Ao longo dos meses de amostragem foram obtidos dados de comprimento da carapaça de 754 exemplares de $P$. defossus, sendo 588 adultos e 166 juvenis. O menor exemplar capturado em campo possuía 3,23 $\mathrm{mm}$ de comprimento e o maior 32,42 $\mathrm{mm}$. Animais com tamanho inferior a $18 \mathrm{~mm}$ de Cc foram considerados como juvenis segundo critérios propostos por Noro et al. (2008), pelo fato de não ser possível diferenciar as gônadas macroscopicamente. Embora o crescimento diferencial entre os sexos seja bem conhecido entre muitos crustáceos, no presente trabalho optou-se por reunir machos e fêmeas para o cálculo da curva de crescimento. Esta decisão metodológica apoiou-se na impossibilidade de distinguir-se, com um mínimo de segurança, o sexo dos animais com base na morfologia externa. Além disso, $P$. defossus é uma espécie hermafrodita protândrica, portanto seqüencial (ver Noro et al. 2008), o que torna a identificação sexual ainda mais insegura.

A figura 1 representa as distribuições de freqüência absoluta, por estação do ano, das classes de comprimento da carapaça (mm) de $P$. defossus. Observa-se a entrada de juvenis na população (recrutamento) na primavera de 2003 e no verão 2005 (provavelmente o recrutamento ocorre na primavera 2004, mas devido à seca não foi possível coletar os juvenis). Os histogramas de freqüência mostram modas bastante nítidas, podendo-se observar três grupos etários a partir da primavera-verão: o primeiro grupo representa a geração de animais juvenis que entraram na população (recrutamento), uma segunda geração de animais com aproximadamente um ano de idade e tamanho aproximado entre $16-18 \mathrm{~mm}$ CC, e a terceira geração, de indivíduos maiores e mais velhos, com idade aproximada de dois anos e com tamanhos superiores a $24 \mathrm{~mm}$ de Cc.

A sucessão de modas escolhida para o cálculo da curva de crescimento foram aquelas que obtiveram o melhor coeficiente de correlação bem como as que ensejaram uma estimativa do comprimento assintótico mais próximo dos dados observados no campo. A tabela I apresenta os valores utilizados para o cálculo dos parâmetros da curva de crescimento. O valor escolhido para representar o comprimento na idade 0 foi de 3,12 $\mathrm{mm}$, visto que este foi o tamanho do menor animal mensurado de uma coorte eclodida em laboratório.

A curva de crescimento em comprimento para os indivíduos de $P$. defossus coletados em campo é descrita pela equação: $\mathrm{Ct}=30,98\left[1-\mathrm{e}^{-0,0026(t+44,86)}\right]$ (Fig. 3). O comprimento assintótico estimado pela curva de crescimento $(30,98 \mathrm{~mm})$ ficou muito próximo do comprimento do maior animal medido em campo (32,42 $\mathrm{mm})$.

A longevidade, calculada pela fórmula inversa de voN BERTALlANFy (1938), foi estimada em 1.219,56 dias, semelhante ao valor calculado pelo método de Pauly (1980), 1.198,7 dias, o que representa uma longevidade de aproximadamente 40 meses (três anos e quatro meses) para a espécie em questão. 

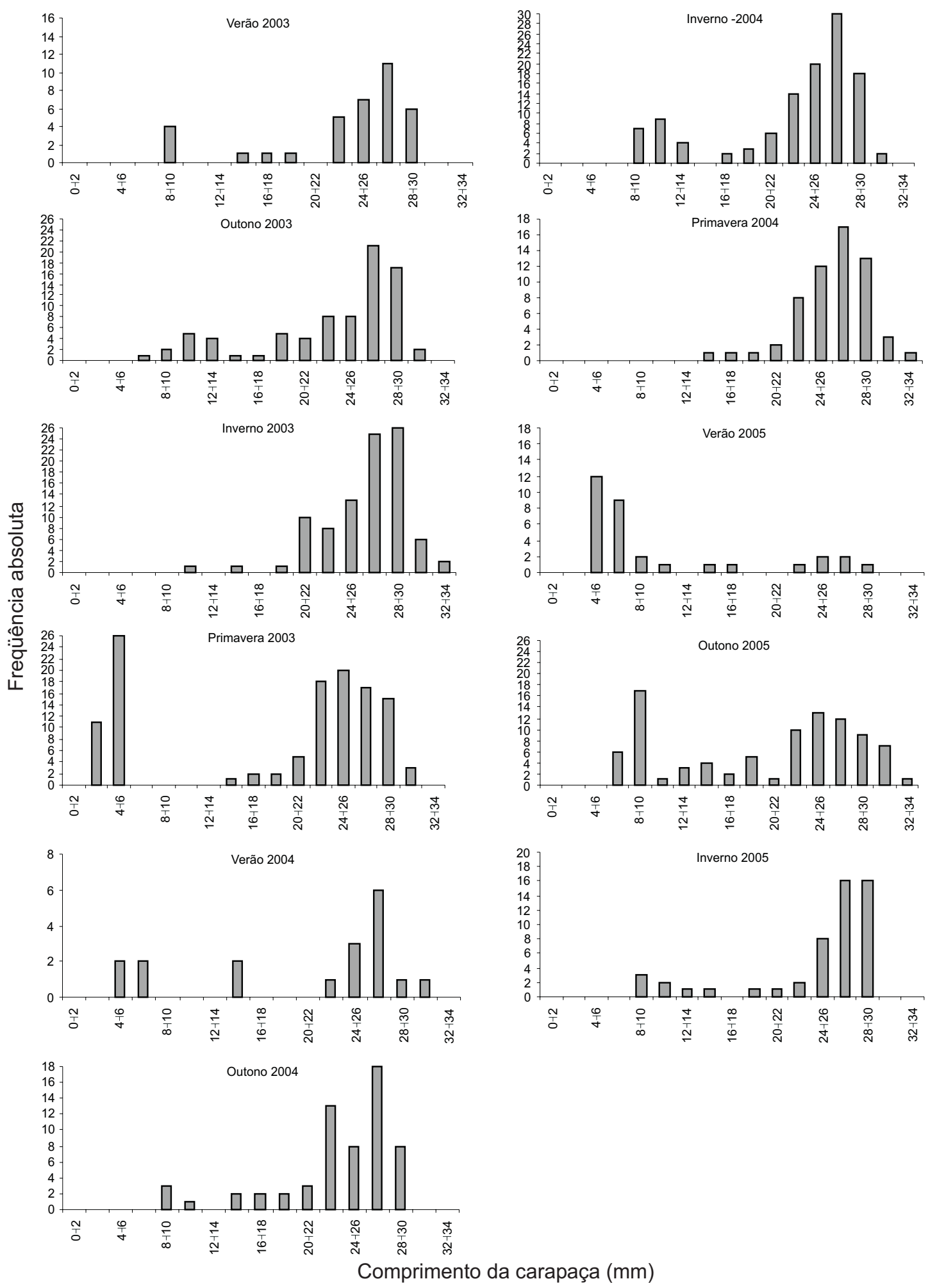

Figura 1. Distribuição das freqüências absolutas sazonais do comprimento da carapaça $(\mathrm{mm})$ de $P$. defossus, coletados no período de janeiro de 2003 a agosto de 2005. 

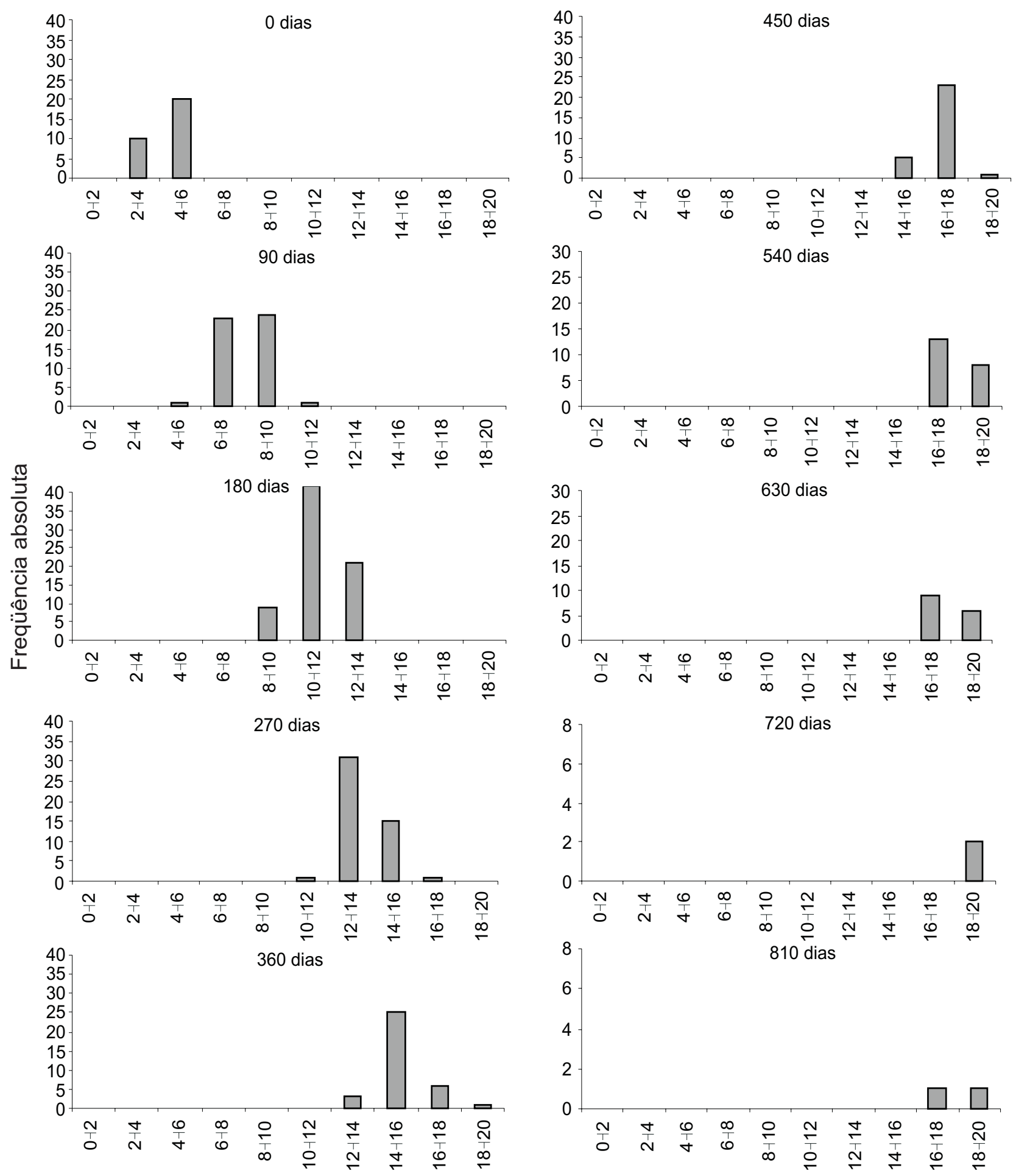

Comprimento da carapaça $(\mathrm{mm})$

Figura 2. Distribuição das freqüências absolutas trimestrais do comprimento da carapaça $(\mathrm{mm})$ dos juvenis de $P$. defossus, cultivados em laboratório no período de dezembro de 2003 a fevereiro de 2006. 


\section{Lagostins eclodidos e cultivados em laboratório}

A figura 2 representa a distribuição de freqüência absoluta das classes de comprimento da carapaça $(\mathrm{mm})$ dos juvenis de $P$. defossus ao longo do ano. A tabela I contém os valores utilizados para o cálculo da curva de crescimento.

Tabela I. Valores das medianas ( $\mathrm{mm}$ ) utilizadas para o cálculo da curva de crescimento de exemplares de $P$. defossus coletados em campo e cultivados em laboratório.

\begin{tabular}{|c|c|c|c|}
\hline \multicolumn{2}{|c|}{$\begin{array}{c}\text { Exemplares coletados } \\
\text { em campo }\end{array}$} & \multicolumn{2}{|c|}{$\begin{array}{l}\text { Juvenis eclodidos } \\
\text { em laboratório }\end{array}$} \\
\hline Idade em dias & Modas & Idade em dias & Modas \\
\hline 0 & 3,12 * & 0 & $3,12^{*}$ \\
\hline 90 & 9,6 & 90 & 9,0 \\
\hline 180 & 12,5 & 180 & 12,37 \\
\hline 360 & 22,2 & 270 & 14,64 \\
\hline 540 & 24,0 & 360 & 16,19 \\
\hline 810 & 26,99 & 450 & 17,99 \\
\hline \multirow[t]{3}{*}{990} & 29,02 & 540 & 18,24 \\
\hline & & 630 & 18,32 \\
\hline & & 810 & 18,71 \\
\hline
\end{tabular}

* Valor referente ao menor animal obtido em laboratório.

A curva de crescimento em comprimento para os juvenis de $P$. defossus cultivados em laboratório é descrita pela equação: $\mathrm{Ct}=19,29$ [1 - $\left.\mathrm{e}^{-0,0048(\mathrm{t}+37,14)}\right]$ (Fig. 3). O valor do comprimento assintótico ficou subestimado, quando comparado com dados de animais em campo, pelo fato de os animais terem sido medidos apenas por 26 meses, sendo que nesta idade o comprimento do único animal que permanecia vivo correspondia ao valor de $17,80 \mathrm{~mm}$.

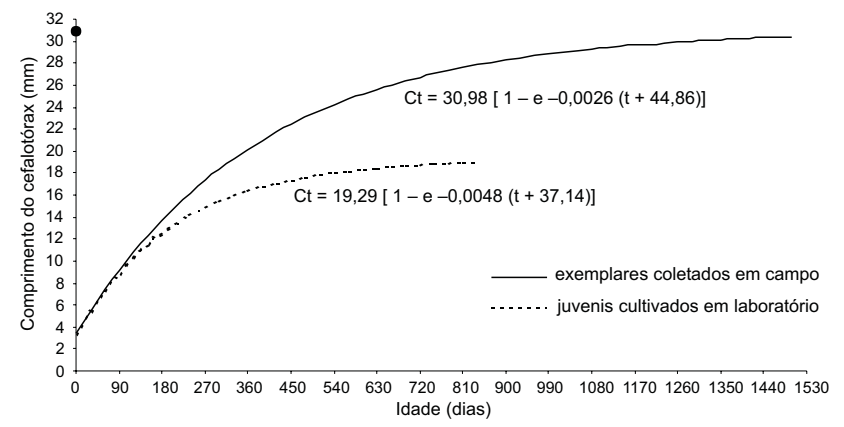

Figura 3. Curva de crescimento em comprimento da carapaça de P. defossus, de exemplares coletados em campo e de juvenis cultivados em laboratório.

\section{DISCUSSÃO}

Os dados do crescimento dos juvenis de $P$. defossus cultivados em laboratório ajudaram na interpretação das medidas obtidas sobre o crescimento dos lagostins em campo, especial- mente na fase inicial. Comparando-se as curvas de crescimento obtidas em campo com a curva dos animais cultivados em laboratório foi possível verificar que, levando em consideração as condições diferenciadas do cultivo, onde a temperatura é constante e a alimentação é distinta, o crescimento em laboratório se mostrou inferior ao crescimento dos animais em campo. Segundo Hartnoll (1983), fatores como alimento e fotoperíodo podem ter efeito em dois componentes essenciais do crescimento, incremento por muda e freqüência da muda. O valor de $\mathrm{k}$ foi superior nos juvenis cultivados em laboratório, mas isto deve-se ao fato do crescimento ser mais acelerado nos animais menores.

Dados de crescimento de espécies de Parastacus, só existem para a P. brasiliensis. Fries (1984) estimou a curva de crescimento para uma coorte nascida em laboratório (animais proveniente do Arroio Dilúvio, Viamão, Rio grande do Sul) $(\mathrm{Ct}=$ $\left.42,894\left[1-\mathrm{e}^{-0,00198(t+48,273)}\right]\right)$, sendo que o valor do comprimento assintótico está um pouco abaixo do valor máximo de 45,4 mm observados por Buckup \& Rossi (1980), em lagostins coletados no meio natural. Na figura 4 é possível observar a diferença entre o crescimento de $P$. defossus e $P$. brasiliensis, ambos cultivados em laboratório. Verifica-se que em condições de cultivo, $P$. defossus apresenta um crescimento muito mais lento, atingindo tamanhos menores que $P$. brasiliensis.

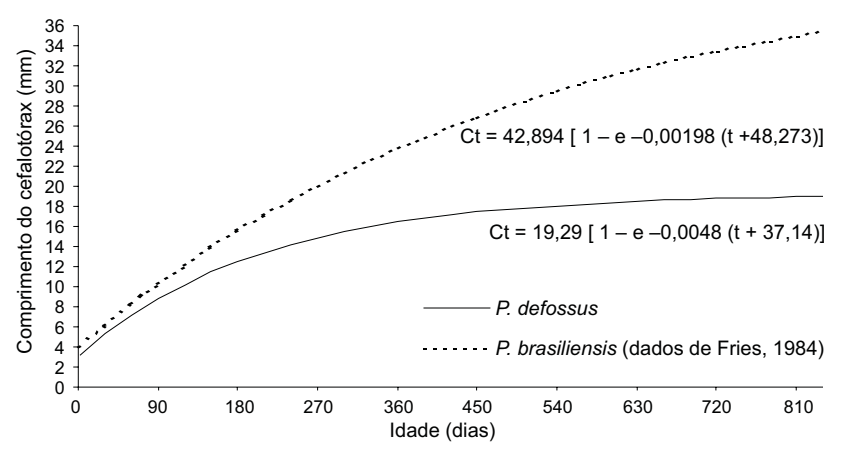

Figura 4. Comparação entre as curvas de crescimento em comprimento da carapaça de juvenis de $P$. defossus e $P$. brasiliensis, segundo dados de Fries (1984), cultivados em laboratório.

FonTOURA \& BUCKUP (1989) calcularam duas curvas de crescimento para uma população de $P$. brasiliensis provenientes do Município de Guaíba, Rio Grande do Sul, a primeira para indivíduos com menos de seis meses de idade $\left(\mathrm{Ct}=17,13\left[1-\mathrm{e}^{-1,940}\right.\right.$ $(\mathrm{t}+0,104)])$ e a segunda para indivíduos com idades superiores a seis meses $\left(\mathrm{Ct}=57,37\left[1-\mathrm{e}^{-0,227(\mathrm{t}+0,487)}\right]\right)$. A necessidade de utilização de duas curvas de crescimento decorreu do fato de que a curva estimada a partir das médias de comprimento dos grupos etários apresentarem uma superestimativa do tamanho do animal ao nascer $\left(\mathrm{L}_{0}\right)$, assim como o valor do $t_{0}$. Os autores comentam sobre a diferença do valor de $\mathrm{k}$ estimado por FriEs (1984), que seria superior ao valor calculado para as condições 
de campo, como ocorreu em $P$. defossus, e que tal fato deve-se provavelmente a fatores de cultivo como temperatura e alimentação. Observa-se que o crescimento de $P$. defossus, em comparação com o crescimento de $P$. brasiliensis, apresenta taxas menores de crescimento, um fator certamente relacionado ao tamanho máximo atingido pela espécie (Fig. 5).

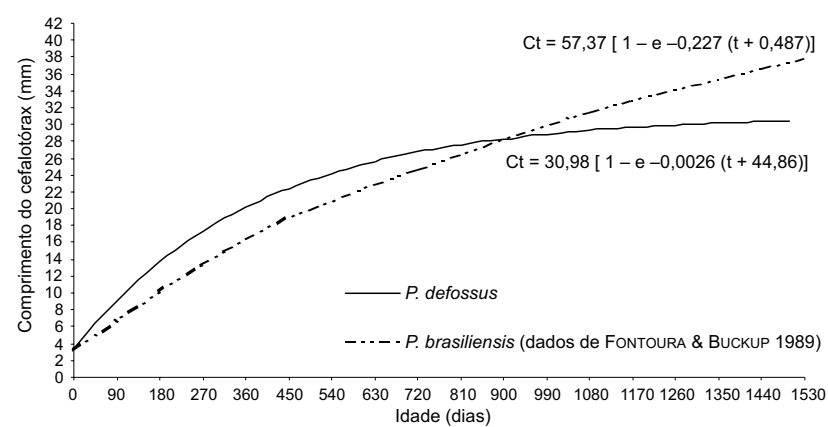

Figura 5. Comparação entre as curvas de crescimento em comprimento da carapaça de $P$. defossus e $P$. brasiliensis, segundo dados de Fontoura \& Buckup (1989), coletados em campo.

A longevidade de $P$. defossus foi estimada em 1198,7 dias ou 40 meses, mostrando-se inferior a longevidade calculada por Fries (1984) para P. brasiliensis, que obteve um valor de 1563 dias ou 52 meses, valor um pouco inferior ao limite de idade estimado por Fontoura \& Buckup (1989), para a mesma espécie, em torno de 6 anos (aproximadamente 72 meses).

Dados sobre o crescimento também existem para espécies de Cambaridae e para Parastacídeos australianos. Fidalgo et al. (2001) estudaram o ciclo de vida do lagostim cambarídeo Procambarus clarkii (Girard, 1852) e observaram um período de recrutamento extenso, com pico em outubro e novembro. A curva de crescimento para a espécie é descrita pela equação: $\mathrm{L}=62,01\left[1-\mathrm{e}^{-0,23(\mathrm{t}+0,11)}\right]$. BEATTY et al . (2005) estudaram o crescimento de Cherax quinquecarinatus (Von Martens, 1898), um lagostim australiano, e obtiveram curvas de crescimento para machos e fêmeas. O valor de $\mathrm{k}$ foi de 0,29 para fêmeas e 0,25 para machos. Apesar do valor de $\mathrm{k}$ ser superior ao que foi estimado em $P$. defossus, o tamanho da espécie com um ano de idade (machos: 18,4 mm e fêmeas: 19,2 $\mathrm{mm}$ ) é semelhante ao encontrado no presente estudo. O tamanho assintótico foi estimado em 59,6 mm para fêmeas e 73,8 mm para machos, valores muito maiores quando comparados com aqueles encontrados para as espécies de Parastacus.

\section{LITERATURA CITADA}

Beatty, S. J.; D. L. Morgan \& S. Howard. 2005. Life History and reproductive biology of the gilgie, Cherax quinquecarinatus, a freshwater crayfish endemic to southwestern Australia. Journal of Crustacean Biology 25 (2): 251-262.
Boos Jr, H.; D. Silva-Castiglioni; K. Schacht; L. Buckup \& G. BondBuckup. 2006. Crescimento de Aegla jarai Bond-Buckup \& Buckup (Crustacea, Anomura, Aeglidae). Revista Brasileira de Zoologia 23 (2): 490-496.

Buckup, L. 1999. Família Parastacidae, p. 319-327. In: L. Buckup \& G. Bond-Buckup (Eds). Os Crustáceos do Rio Grande do Sul. Porto Alegre, UFRGS, 503p.

Buckup, L. \& A. Rossi. 1980. O gênero Parastacus no Brasil (Crustacea, Decapoda, Parastacidae). Revista Brasileira de Biologia 40 (4): 663-681.

Bueno, A.A.P.; G. Bond-Buckup \& L. Buckup. 2000. Crescimento de Aegla platensis Schmitt em ambiente natural (Crustacea, Decapoda, Aeglidae). Revista brasileira de Zoologia 17 (1): 51-60.

Castiglioni, D.S.; D. Silva-Castiglioni \& M.L.N. Fransozo. 2004. Somatic growth of the fiddler crab Uca rapax (Smith, 1870) (Brachyura, Ocypodidae) from two subtropical mangroves. Universidad y Ciencia 20 (39): 15-22.

Díncao, F.; M.L. Ruffino; K.G. Silva; A.C. Braga \& L.H.C. MarQUES. 1993. Crescimento de Chasmagnatus granulata Dana, 1851 em um marisma de um estuário da Lagoa dos Patos, Rs (Decapoda, Grapsidae). Revista Brasileira de Biologia 53 (4): 637-643.

Dumont, L.F.C. \& F. D'InCAO. 2004. Growth and reproductive pattern of the caridean shrimp Palaemonetes argentinus Nobili, 1901 (Decapoda: Palaemonidae) in the south of Rio Grande do Sul, Brazil. Nauplius 12 (2): 151-163.

Ferreira, L.S. \& F. D'Incao. 2008. Crescimento de Callinectes sapidus (Crustacea, Decapoda, Portunidae) no estuário da laguna dos Patos, RS, Brasil. Iheringia, Série Zoologia, 98: 70-77.

Fidalgo, M.L.; A.P. Carvalho \& P. Santos. 2001. Population dynamics of the red swamp crayfish Procambarus clarkii (Girard, 1852) from the aveiro region, Portugal (Decapoda, Cambaridae). Crustaceana 74 (4): 369-375.

Fonteles-Filho, A.A. 1987. Recursos pesqueiros, biologia e dinâmica populacional. Fortaleza, Imprensa Oficial de Ceará, 296p.

Fontoura, N.F. \& L. Buckup. 1989. O crescimento de Parastacus brasiliensis (von Martens, 1869) (Crustacea, Decapoda, Parastacidae). Revista Brasileira de Biologia 49 (4): 897-909.

Fries, B.G. 1984. Observações sobre o lagostin de água doce Parastacus brasiliensis (von Martens, 1869) em condições de cultivo experimental em laboratório (Crustacea, Decapoda, Parastacidae). Revista Brasileira de Biologia 44 (4): 409-416. HartNoll, R.G. 1983. Growth, p. 253-321. In: D.E. Bliss (Ed). The biology of Crustacea: embriology, morphology and genetics. New York, Academic Press, X+550p.

Noro, C.K. \& L. Buckup. 2003. O crescimento de Aegla leptodactyla Buckup \& Rossi (Crustacea, Anomura, Aeglidae). Revista brasileira de Zoologia 20 (2): 191-198.

Noro, C.K.; L.S. López-Grego \& L. Buckup. 2008. Gonad morphology and type of sexuality in Parastacus defossus Faxon 1898, a burrowing, intersexed crayfish from southern Brazil (Deca- 
poda: Parastacidae). Acta Zoológica 89: 59-67.

PAULY, D. 1980. On the interrelationships between natural mortality, growth parameters and mean environmental temperature in 175 fish stocks. Journal of Conservation and International Exploration, 39 (2): 175-192.

Pinheiro, M.A.A. \& F.G. TAdDeI. 2005. Crescimento do caranguejo de água doce, Dilocarcinus pagei Stimpsin (Crustacea, Brachyura, Trichodactylidae). Revista Brasileira de Zoologia 22 (3): 522-528.

Sampaio, C.M \& W.C. Valenti. 1996. Growth of Macrobrachium rosembergii in semi-intensive culture in Brazil. Journal of World Aquaculture Society 27 (3): 353-358.

SANTOS, E.P. 1978. Dinâmica de populações aplicada à pesca e piscicultura. São Paulo, Hucitec, Edusp, 129p.
Silva-Castiglioni D.; D.F.Barcelos \& S. Santos. 2006. Crescimento de Aegla longirostri Bond-Buckup \& Buckup, 1994 (Crustacea, Anomura, Aeglidae). Revista brasileira de Zoologia 23: 408-413.

Valenti, W.C.; J.T.C. De Mello \& V.L. LobÃo. 1987. Crescimento de Macrobrachium acanthurus (Wiegman 1836) do Rio Ribeira do Iguape (Crustacea, Decapoda, Palaemonmidae). Revista Brasileira de Biologia 47 (3): 349-355.

VON Bertalanffy, L. 1938. A quantitative theory of organic growth. Human Biology 10 (2): 181-213.

Zolessi, L.C. \& M.E. PhilipPI. 1995. Lista sistematica de Decapoda del Uruguay (Arthropoda: Crustacea). Comunicationes Zoologicas del Museo de Historia Natural de Montevideo 12: 1-24.

Submitted: 26.VI.2008; Accepted: 16.III.2009.

Editorial responsibility: Maria Lúcia Negreiros-Fransozo 